\title{
Fracture of complex metallic alloys: An atomistic study of model systems
}

\author{
Frohmut Rösch and Hans-Rainer Trebin \\ Institut für Theoretische und Angewandte Physik, Universität Stuttgart, \\ Pfaffenwaldring 57, 70550 Stuttgart, Germany \\ Peter Gumbsch \\ Institut für Zuverlässigkeit von Bauteilen und Systemen, \\ Universität Karlsruhe, Kaiserstr. 12, 76131 Karlsruhe, Germany \\ Fraunhofer Institut für Werkstoffmechanik, Wöhlerstr. 11, 79108 Freiburg, Germany
}

\begin{abstract}
Molecular dynamics simulations of crack propagation are performed for two extreme cases of complex metallic alloys (CMAs): In a model quasicrystal the structure is determined by clusters of atoms, whereas the model C15 Laves phase is a simple periodic stacking of a unit cell. The simulations reveal that the basic building units of the structures also govern their fracture behaviour. Atoms in the Laves phase play a comparable role to the clusters in the quasicrystal. Although the latter are not rigid units, they have to be regarded as significant physical entities.
\end{abstract}

\section{Introduction}

Complex metallic alloys are intermetallic compounds with large unit cells containing from tens up to thousands of atoms. Often, distinct local arrangements of atoms - clusters - can be viewed as building units. Both, the cluster diameter and the lattice constant imply length scales which should be reflected in physical properties. CMAs frequently combine interesting properties like high melting point, high temperature strength, and low density. However, possible applications are often limited by extreme brittleness at low or ambient temperature. To enlighten the role of clusters and periodicity in fracture, we perform molecular dynamics simulations of two extreme cases of CMAs: An icosahedral quasicrystal and a C15 Laves phase. As we are interested in the general qualitative features of the structures, we use three-dimensional model systems consisting of about five million atoms and model potentials (Lennard-Jones). This deliberate choice in the past often helped to reveal fundamental aspects of fracture (see e.g. Abraham (2003)). The quasicrystal can be viewed as a CMA with an infinitely large unit cell, such that no periodicity is present and clusters are the main feature of the structure. On the other hand, the C15 Laves phase has 24 atoms in the cubic unit cell and no clusters. The structure of the Laves phases is determined by periodicity but already quite complex, such that complicated deformation mechanisms might emerge (see e.g. Chisholm et al. (2005)). 


\section{Models and method}

The three-dimensional model quasicrystal used in our numerical experiments has been proposed by Henley and Elser (1986) as a structure model for icosahedral ( $\mathrm{Al}, \mathrm{Zn})_{63} \mathrm{Mg}_{37}$. It is built up from the prolate and oblate rhombohedra of the three-dimensional Penrose tiling. As we do not distinguish between $\mathrm{Al}$ and $\mathrm{Zn}$ type atoms, we term its decoration icosahedral binary model (for details see e.g. Rösch et al. (2005)). In the upcoming figures the $\mathrm{Al}$ and $\mathrm{Zn}$ type atoms (A atoms) are displayed as grey (online: red) balls, whereas $\mathrm{Mg}$ type atoms (B atoms) are shown in black (online: blue). The shortest distance between two A atoms is denoted $r_{0}$ which corresponds to about $2.5 \AA$. Inherent in the structure are Bergman-type clusters, which also can be viewed as basic building units.

As no reliable "realistic" effective potentials are available for the fracture of CMAs, the interactions are modelled by simple Lennard-Jones pair potentials (see Sec. 1, Rösch et al. (2004), and Rösch et al. (2005)). These potentials keep the model stable even under strong deformations or introduction of point defects and have been used in our group together with the icosahedral binary model to simulate dislocation motion (Schaaf et al. (2003)) and even shock waves $(\operatorname{Roth}(2005))$. Very similar potentials have shown to stabilize the icosahedral atomic structure (Roth et al. (1995)). By the choice of these model potentials and model structures we qualitatively probe the influence of structural aspects of the investigated compounds without being specific to a special kind of material. Model potentials are often used in fracture simulations and have led to useful insight into fundamental mechanisms (see e.g. Abraham (2003)). The minimum of the Lennard-Jones potential for the interactions between atoms of different kind is set to twice the value of that for atoms of the same type. However, the conclusions drawn from our simulations remain essentially unaffected by setting all binding energies equal, which again indicates that we are mainly probing structural effects.

A fundamental building unit of the simulated quasicrystal - the prolate rhombohedron in a slightly deformed way forms the cubic C15 Laves phase $\mathrm{A}_{2} \mathrm{~B}$ by periodic arrangement. But in this structure no clusters of the quasicrystal are present. Because of the close structural relationship of the C15 Laves phase to the quasicrystal model, we use the same model potentials.

Our samples have dimensions of approximately $450 r_{0} \times 150 r_{0} \times 70 r_{0}$ and contain about five million atoms. Periodic boundary conditions are applied in the direction parallel to the crack front. To simulate mode I fracture, we first determine potential cleavage planes. According

to Griffith (1921) crack propagation becomes possible, when the elastic energy is sufficient to generate two new fracture surfaces. Thus, potential cleavage planes should be those with low surface energies. In the quasicrystal these are specific twofold and fivefold planes (see Rösch et al. (2005)). There atomically sharp seed cracks are inserted. The samples are uniaxially strained perpendicular to the crack plane up to the Griffith load and then relaxed to obtain the displacement field of the stable crack at zero temperature. Subsequently, a temperature of about $10^{-4}$ of the melting temperature is applied. The sample is further loaded by linear scaling of the corresponding displacement field for this temperature. The response of the system then is monitored by molecular dynamics techniques. The radiation emitted by the propagating crack is damped away outside an elliptical region to prevent reflections (see Rösch et al. (2005) and Gumbsch et al. (1997)). 


\section{Results and discussion}

In the model quasicrystal we investigated how clusters and the plane structure influence crack propagation. A paper on this detailed study has recently been published (see Rösch et al. (2005)), where brittle fracture without any crack tip plasticity was reported. The following results from that paper indicate that the clusters determine the brittle cleavage fracture of the model quasicrystal: First, circumvention or intersection of clusters slows down the cracks. Second, the fracture surfaces show characteristic height variations giving rise to an overall roughness on the cluster scale (see Fig. 1, left). Lines along which the clusters are located are also visible in the height profiles. Third, the crack intersects fewer clusters than a planar cut with low surface energy does (see Fig. 2). Another observation of the simulations is that the plane structure also influences fracture. Cracks located perpendicular to twofold and fivefold axes fluctuate about a constant height. Thus, the roughness of the crack surfaces can be assigned to the clusters, whereas constant average heights of the fracture surfaces reflect the plane structure of the quasicrystal.

Now we compare simulation results of the quasicrystal to those of the C15 Laves phase. For this structure we also observe brittle failure. But the fracture surfaces at low loads - if at all - only are rough on an atomic scale. This becomes apparent from Figs. 1 and 3.

In Fig. 3 only atoms near a (111) fracture surface are displayed. The seed crack shown on the top propagated in [2 $\overline{1} \overline{1}]$ direction. The material perfectly cleaved (Fig. 3 , bottom), which resulted in smooth fracture surfaces.

In Fig. 1 sections of geometrically scanned fracture surfaces of the icosahedral model quasicrystal (left) and of the C15 model Laves phase (middle, right) are compared. The cleavage planes are located perpendicular to a twofold (left) and a [010] axis (middle, right). The crack propagated along a twofold (left) and a [101] axis (middle, right). The colour coding in the left image is adjusted to the cluster diameter (online: from blue to red). When colour coded like the quasicrystal, the fracture surfaces of the C15 Laves phase lack any roughness (middle, online: only green). After adjusting the colour coding, atomic rows become visible (right). So, fracture surfaces of the quasicrystal are rough on the cluster scale, whereas those of the Laves phase only are rough on an atomic scale.

Thus, atoms in the Laves phase play a comparable role to the clusters in the quasicrystal they determine the overall roughness of the fracture surfaces. The atomistic view of cleavage on a (011) plane in Fig. 4 reveals an interesting effect ${ }^{1}$. If the seed crack there would be continued, the lines would terminate the upper and lower halves of the sample. However, as can be seen in the time sequence of Fig. 4, this is not the case: The dynamic crack instead takes a zig-zag like route. Entire atomic rows alternately move upwards and downwards. This leads to rather symmetric upper and lower fracture surfaces, the creation of which also requires a comparable amount of energy. This rather symmetrical creation of fracture surfaces is favoured, even though a planar cut would lead to surfaces with lower total energy. Thus, the actual fracture path cannot be predicted by a simple energy criterion. Such a behaviour also was observed in B2 NiAl (see e.g. Gumbsch (2001)) and is a consequence of lattice trapping (Thomson et al. (1971)), which - similar to the Peierls barrier for dislocation motion - allows overloads for cracks that do not result in crack propagation. The increased load for a crack to propagate will therefore not necessarily lead to fracture surfaces of lowest energy. The discrete nature of matter is responsible for these observations. The fracture path is strongly

\footnotetext{
${ }^{1} \mathrm{~A}$ similar behaviour is observed for the orientation shown in Fig. 1 (middle, right).
} 
influenced by the arrangement of atoms near the crack front, which depends on the initial cleavage plane as well as the crack propagation direction.

\section{Conclusions and outlook}

In conclusion, the simulation results of the two extreme cases of model CMAs indicate that the basic building units of the structures govern also their physical properties. The role of atoms in the Laves phase is - from a certain point of view - played by the clusters in the quasicrystal. Although these are not rigid units, they are significant physical entities.

To further enlighten the fracture processes in the C15 Laves phase, we currently perform simulations with force-matched (Ercolessi and Adams (1994), Brommer and Gähler (2006)) effective embedded atom method potentials for $\mathrm{NbCr}_{2}$ (Rösch et al. (2006)). Although we expect that the overall qualitative behaviour (e.g. the roughness of the fracture surfaces) is already represented well by our simple model potentials, results certainly will differ quantitatively for diverse materials, i.e. interactions. Especially, the lattice trapping mentioned above strongly depends on the potentials used.

Future studies will concentrate on material specific simulations on systems of CMAs, in which compounds with very different unit cell sizes and local arrangements exist. New effects are expected e.g. when the size of the unit cell and cluster diameters become comparable.

\section{Acknowledgement}

Financial support from the Deutsche Forschungsgemeinschaft under contract number TR $154 / 20-1$ is gratefully acknowledged.

\section{References}

F. F. Abraham, Advances in Physics 52 (8), 727 (2003).

M. F. Chisholm, S. Kumar, and P. Hazzledine, Science 307, 701 (2005).

C. L. Henley and V. Elser, Phil. Mag. B 53 (3), L59 (1986).

F. Rösch, Ch. Rudhart, J. Roth, H.-R. Trebin, and P. Gumbsch, Phys. Rev. B 72, 014128 (2005).

F. Rösch, Ch. Rudhart, P. Gumbsch, and H.-R. Trebin, Mat. Res. Soc. Symp. Proc. 805, LL9.3 (2004).

G. D. Schaaf, J. Roth, and H.-R. Trebin, Phil. Mag. 83 (21), 2449 (2003).

J. Roth, Phys. Rev. B 71, 064102 (2005).

J. W. Roth, R. Schilling, and H.-R. Trebin, Phys. Rev. B 51 (22), 15833 (1995).

A. A. Griffith, Philos. Trans. R. Soc. Lond. Ser. A 221, 163 (1921).

P. Gumbsch, S. J. Zhou, and B. L. Holian, Phys. Rev. B 55 (6), 3445 (1997).

P. Gumbsch, Mater. Sci. Eng. A 319-321, 1 (2001).

R. Thomson, C. Hsieh, and V. Rana, J. Appl. Phys. 42 (8), 3154 (1971).

F. Ercolessi and J. B. Adams, Europhys. Lett. 26 (8), 583 (1994). 
P. Brommer and F. Gähler, Phil. Mag. 86 (6-8), 753 (2006).

F. Rösch, H.-R. Trebin, and P. Gumbsch, Int. J. Fracture 139 (3-4), 517 (2006).

\section{Figures}
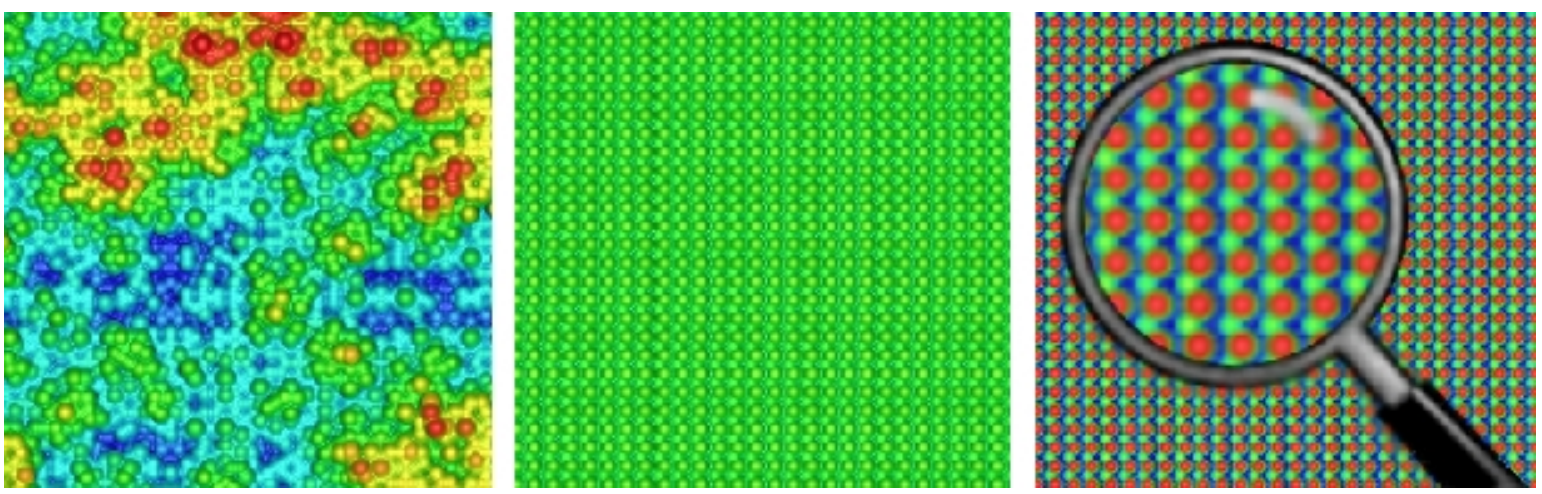

Figure 1: Sections of typical fracture surfaces of an icosahedral model quasicrystal (left) and of a C15 model Laves phase (middle, right). The colour coding in the left and middle picture is adjusted to the cluster diameter (online: from blue to red). For details see text. The side length of the squares is about $14 \mathrm{~nm}$.

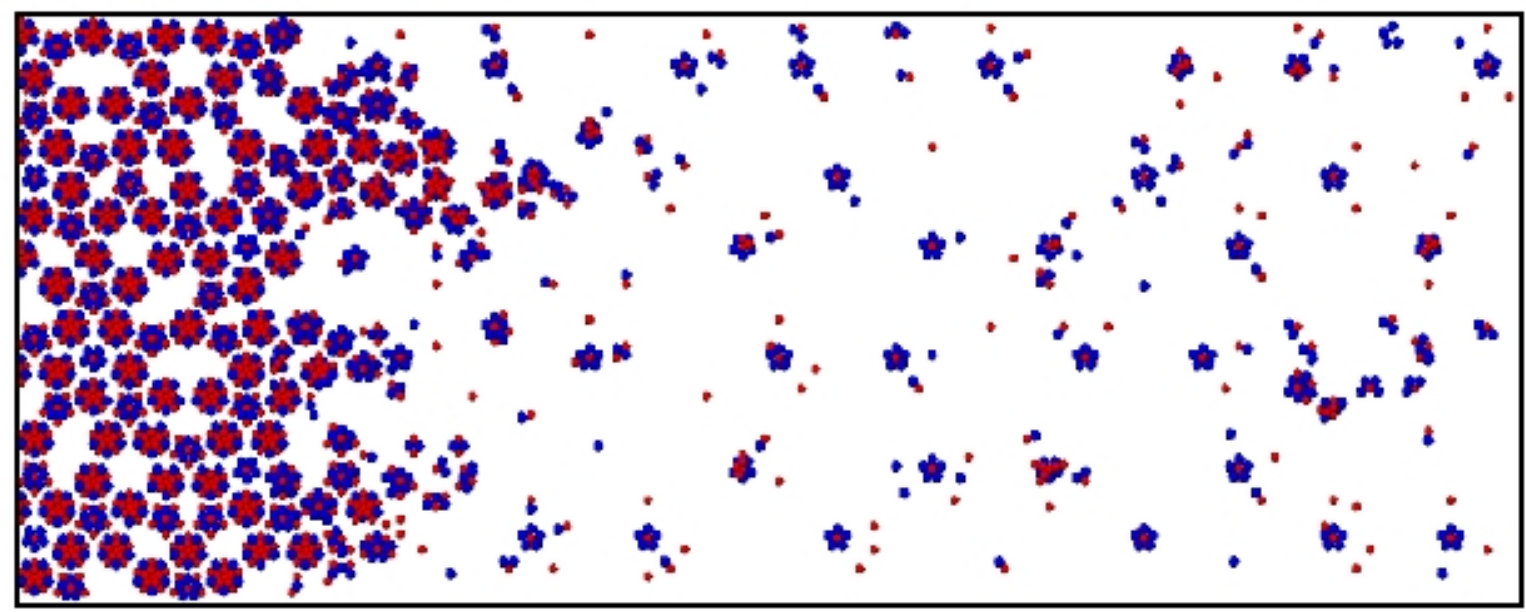

Figure 2: Clusters cut by the crack in a model quasicrystal: Only the smaller parts of those clusters are displayed that were divided by the crack. Obviously, the dynamic crack (right) intersects fewer clusters than the low energy seed crack (left). The cleavage plane is located perpendicular to a fivefold axis, the crack propagated in twofold direction. 

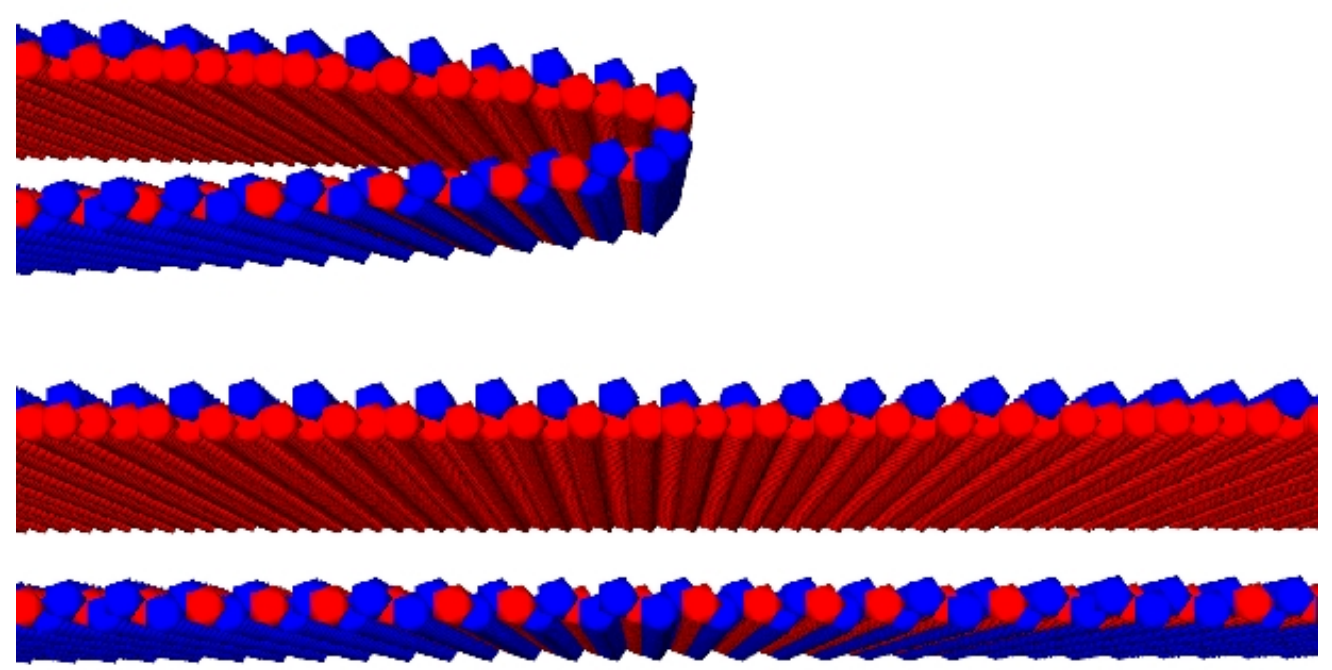

Figure 3: View inside a crack of the $\mathrm{C} 15$ model Laves phase. Perfect brittle cleavage fracture is observed.

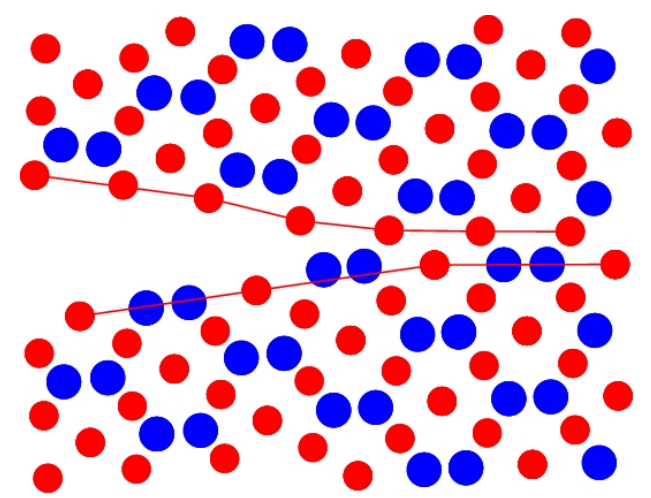

a)

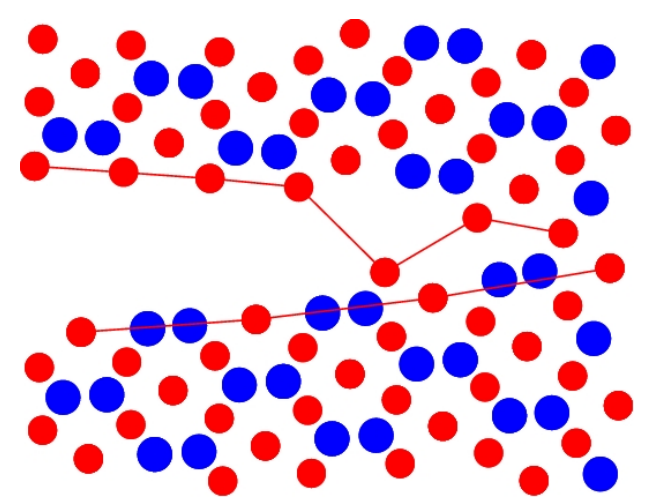

c)

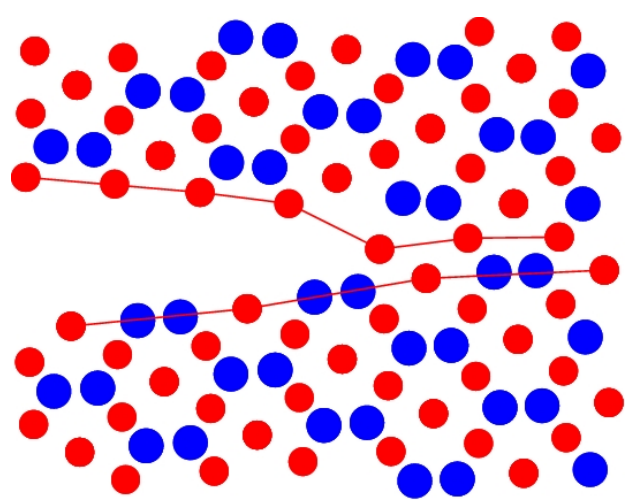

b)

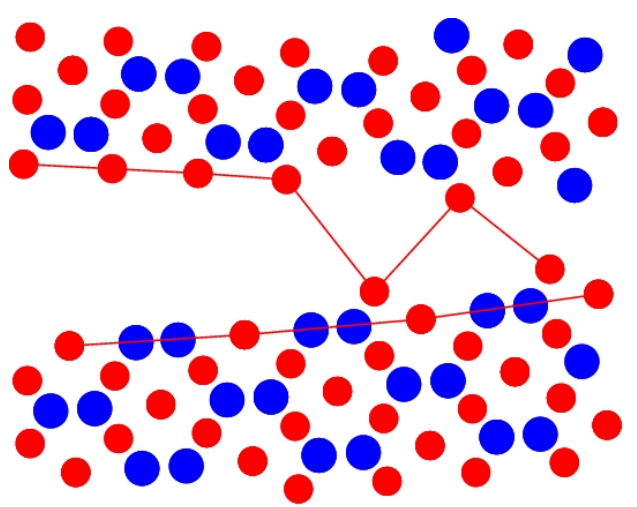

d)

Figure 4: Fracture of a C15 model Laves phase: Atomic configurations in the vicinity of a propagating crack (time sequence). The fracture surface is located perpendicular to the [011] direction, the crack propagates along the [100] direction (from left to right). 